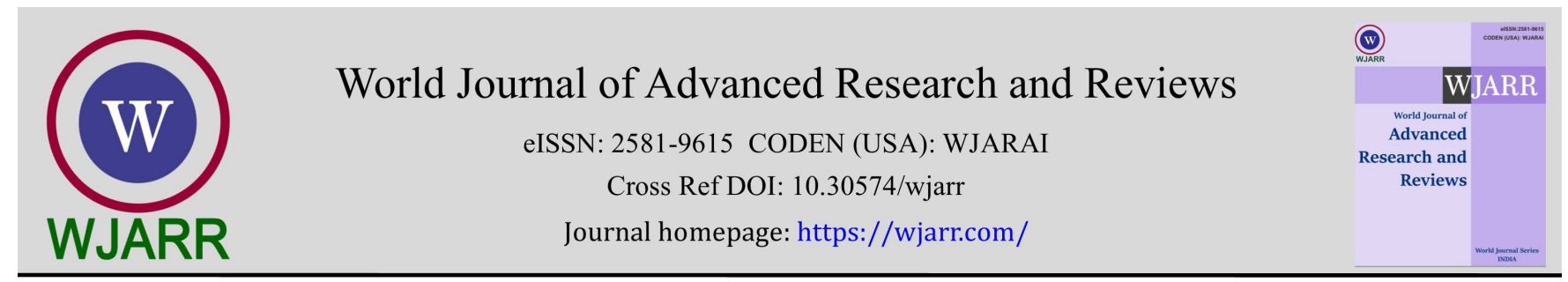

(REVIEW ARTICLE)

\title{
How obesity influence psoriasis severity in developing countries
}

\author{
Mohammad Samiul Huq ${ }^{1,{ }^{*}}$, Abu Hena Chowdhury ${ }^{2}$ and Saleheen Huq ${ }^{3}$ \\ ${ }^{1}$ Psoriasis Awareness Club Bangladesh, Square Hospital Ltd, Dhaka, Bangladesh. \\ 2 Bangabandhu Shaikh Mujib Medical University, Dhaka, Bangladesh. \\ ${ }^{3}$ Peterborough city hospital, Bretton Gate, Peterborough, UK.
}

World Journal of Advanced Research and Reviews, 2021, 11(01), 176-182

Publication history: Received on 18 June 2021; revised on 22 July 2021; accepted on 24 July 2021

Article DOI: https://doi.org/10.30574/wjarr.2021.11.1.0336

\begin{abstract}
Psoriasis is a common chronic inflammatory skin disease with a complex pathogenesis consisting of a genetic component, immune dysfunction, and environmental factors. It is associated with numerous comorbidities including psoriatic arthritis, cardiovascular disease, metabolic syndrome, and obesity. The dietary habits in industrialized nations promote high-fat, high-salt, and high-sugar diets with excess caloric intake resulting in an obesity epidemic. Metaanalyses have shown that (1) genetically higher BMI increased the odds of psoriasis occurrence; (2) obesity is associated with higher incidence and prevalence of psoriasis as well as psoriasis severity; (3) obesity is associated with lower efficacy to anti-TNF agents and may predict biologic treatment discontinuation; and (4) weight loss through diet and physical exercise may improve pre-existing psoriasis. Obesity is one of the major public health concerns due to its upward trend in both developing and developed countries. In developing countries, the consequence of obesity not only affects health but also creates a burden on individual and national healthcare budgets. The prevalence of overweight and obesity in developing countries is increasing faster than the world average. This fast-paced growth has been accompanied by notable increases in the burden of non-communicable diseases (NCDs). While obesity is gradually increasing in developing countries, where NCD is yet to implement. If proper NCD program is not implement in right time, severity of psoriasis will increase with increase prevalence of obesity.
\end{abstract}

Keywords: Obesity; Psoriasis; Severity; Developing countries

\section{Introduction}

Psoriasis is a common chronic inflammatory skin disease with a complex pathogenesis consisting of a genetic component, immune dysfunction, and environmental factors. It is associated with numerous comorbidities including psoriatic arthritis, cardiovascular disease, metabolic syndrome, and obesity. Evidence suggests that obesity is a risk factor for incident psoriasis, aggravates existing psoriasis, and that weight reduction may improve the severity of psoriasis in overweight individuals.

In recent decades, the prevalence of immune-mediated diseases in industrialized nations has been increasing, and also, there is evidence that the epidemiology of psoriasis seems to be changing [1, 2]. For example, a 2009 US study demonstrated that the incidence of psoriasis among adults had almost doubled between the 1970 s and 2000 [3].

The dietary habits in industrialized nations promote high-fat, high-salt, and high-sugar diets with excess caloric intake resulting in an obesity epidemic $[4,5]$. Obesity is a chronic condition characterized by excess body weight due to increased energy deposits stored as body fat. When the equilibrium of food intake and energy expenditure is disturbed, regardless of the cause, this will result in either obesity or low body weight [6].

\footnotetext{
${ }^{*}$ Corresponding author: Mohammad Samiul Huq

Psoriasis Awareness Club Bangladesh, Square Hospital Ltd, Dhaka, Bangladesh.
} 
One of the first reports about the relationship between psoriasis and obesity came from Sweden in a study including 159,200 individuals who were followed up over a 10-year period [7]. In the study, the authors found an association between women with psoriasis and obesity.

Obesity is one of the major public health concerns due to its upward trend in both developing and developed countries $[8,9]$. According to the World Health Organization (WHO), about 39\% of adults aged $\geq 18$ years were overweight and $13 \%$ of adults were obese in the world in 2016 [10]. Based on the WHO report, 1 in 3 of the world's adult population is overweight and 1 in 10 is obese. Undernutrition is more frequent in developing countries, however, over the past two decades, overweight and obesity are increasing rapidly in low to middle-income countries alongside undernutrition [11].

In developing countries, the consequence of obesity not only affects health but also creates a burden on individual and national healthcare budgets [12]. Bangladesh is a low-income and agro-based country where a major percentage of the national population (72\%) lives in rural areas [12]. The country is currently growing at an annual rate of around $1 \%$. Bangladesh reaches a population of 166 million in 2021, and will be 186 million by 2030 [15]. Over the past two decades, Bangladesh has experienced a rapid epidemiological transition from communicable diseases to non-communicable diseases $[13,14]$. Rapid urbanization and industrialization also contribute to the increased prevalence of obesity in the Bangladeshi population.

\section{Hypothesis of linking Obesity and Psoriasis}

\subsection{Autoimmunity and Obesity}

The possible link between autoimmunity and obesity has become even more relevant since the discovery that white adipose tissue is not just an inert energy storage tissue. It is now known that adipose tissue is an essential endocrine organ secreting a wide range of soluble mediators involved in immunity, inflammation, and metabolic and appetite regulation [16,17]. The soluble mediators (Fig. 1) released from white adipose tissue possess pro-inflammatory actions and contribute to the low-grade inflammatory state in obese individuals [16]. This pro-inflammatory state generated by obesity may be a key factor in the association between adiposity and inflammatory/autoimmune diseases which include rheumatoid arthritis, systemic lupus erythematosus, inflammatory bowel disease, multiple sclerosis, diabetes mellitus, hidradenitis suppurativa, and psoriasis $[18,19]$.

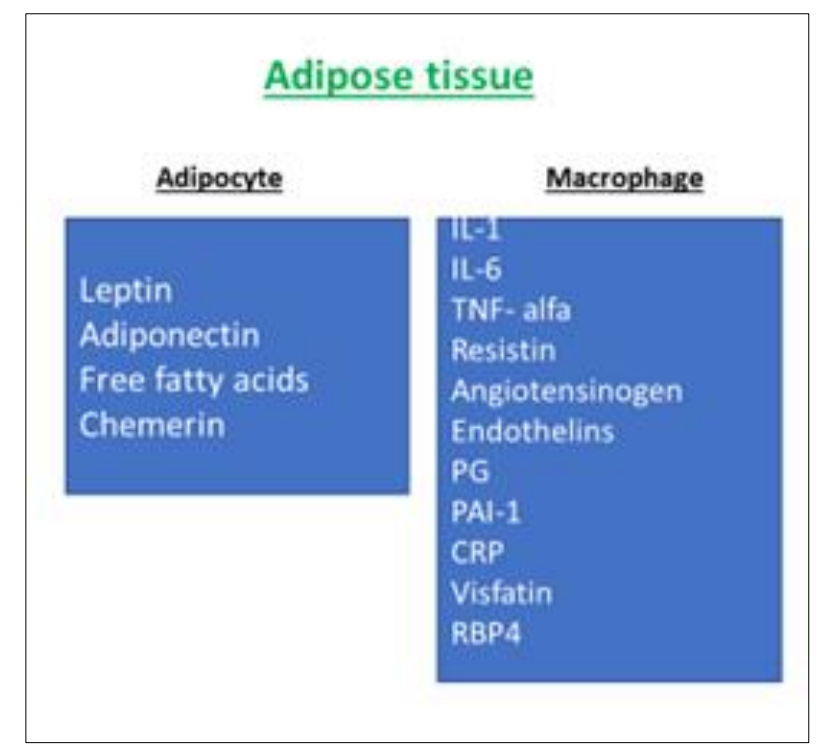

Figure 1 Mediators secreted from Adipose tissue

\subsection{Habitual and behavioral Linking of Psoriasis and Obesity}

Numerous mechanisms may be involved in explaining the link between psoriasis and obesity. Patients with psoriasis may have a higher risk of social isolation, poor eating habits, depression, decreased physical activity, and increased alcohol consumption. Along those lines, Zamboni [20] showed that patients with psoriasis consumed significantly fatter, 
saturated fat, and alcohol compared to people without psoriasis. Also, obese patients with psoriasis exercise less than obese individuals without psoriasis [21].

\subsection{Inflammatory mediators linking}

Recently it has been demonstrated that adiposity may lead to induction of T-helper 17 cells (Th17). Th17 cells secrete IL-17 and are recognized for the involvement in the pathogenesis of autoimmune diseases including psoriasis [22]. Psoriasis is characterized by proliferation of Th1, Th17, and Th22 cells resulting in the production of the proinflammatory mediator's interferon- $\gamma$, TNF- $\alpha$, IL-6, and IL-22 [23, 24]. Therefore, TNF- $\alpha$ and IL- 6 secreted by adipose tissue may contribute to the inflammatory state in psoriasis [25]. With regard to leptin and resistin, several studies have found high levels of both of these adipokines in obese individuals with psoriasis and that plasma concentrations correlated with the severity of psoriasis [24, 26, 27, 28]. Also, it has been demonstrated that resistin levels decreased under treatment. A recent meta-analysis including 11 studies showed that patients with psoriasis have higher levels of leptin compared to persons without psoriasis [24]. Both resistin and leptin promote the secretion of TNF- $\alpha$ and IL-8 which are involved in the pathogenesis of psoriasis. Also, tissue levels of leptin are increased in the skin of patients with psoriasis, and here it stimulates secretion of pro-inflammatory cytokines in vitro [29, 30].

\subsection{Genetic link}

At this point, it is reasonable to wonder whether perhaps there may be a common genetic predisposition for developing both psoriasis and obesity or through which mechanisms the presence of obesity might predispose to psoriasis and vice versa. We know that HLA-Cw6, a major psoriasis susceptibility gene, is also associated with obesity. Obese patients with the HLA-Cw6 gene were 35 times more likely to develop psoriasis than patients of normal weight without the HLA-Cw6 gene [31]. In normal-weight individuals with HLA-Cw6 positivity the risk of psoriasis was 8.33 times greater than for persons with HLA-Cw6 negativity [31]. Studies have shown that psoriasis is mainly associated with central adiposity reflected in an increased waist-to-hip ratio [31]. Since it is clear that psoriasis and obesity may coexist, it is possible that there is an interaction between the 2 diseases, and therefore adipokines may play a regulatory and shared role.

\subsection{Epidemiological evidence}

Epidemiological studies suggested that patients with psoriasis, compared with the general population, have higher prevalence and incidence of obesity [32]. A meta-analysis of 16 observational studies found that the pooled odds ratio (OR) for obesity among patients with psoriasis was 1.66 (95\% confidence interval [CI] 1.46-1.89) compared with those without psoriasis [32].

The relationship between obesity and psoriasis has been demonstrated in animal studies. Employing an obese mouse model with psoriasiform dermatitis induced by imiquimod, it was shown that obesity may acutely aggravate the severity of psoriasiform dermatitis in mice [33]. Furthermore, mice expressed greater levels of psoriasis mediators, such as IL22 and IL-17A and its downstream protein regenerating islet-derived $3 \gamma$, which has been implicated in psoriatic epidermal hyperplasia as a critical mediator [33]. These findings suggest that obesity may exacerbate psoriasiform dermatitis through upregulation of pro-inflammatory cytokines.

A number of meta-analyses have depicted an important bidirectional association between psoriasis and comorbid obesity, with growing evidence showing that obesity may be a predisposing factor for psoriasis while psoriasis patients present higher prevalence and incidence of obesity.

Based upon the fact that diet with high caloric value, sedentary lifestyle, and emotional stress are some of the modifiable shared risk factors in both obesity and psoriasis, the importance of a holistic approach for obese psoriatic patients becomes evident [34]. Particularly, weight loss, especially through education on healthy diet, and physical activity are of paramount importance in psoriasis with comorbid obesity [35].

Treating obese psoriatic patients presents many challenges and should be approached in a holistic and individualized manner. Lifestyle advice and weight loss should be at the center of therapeutic management helping both the psoriatic patient and the efficacy of the pharmacological treatment. Great care should also be given when choosing the right treatment option for these individuals taking into account their metabolic profile.

Recent meta-analyses [36] have shown that (1) genetically higher BMI increased the odds of psoriasis occurrence; (2) obesity is associated with higher incidence and prevalence of psoriasis as well as psoriasis severity; (3) obesity is associated with lower efficacy to anti-TNF agents and may predict biologic treatment discontinuation; and (4) weight loss through diet and physical exercise may improve pre-existing psoriasis and prevent from de novo psoriasis. 


\section{NCD program yet to introduce in developing countries}

As with many high-resourced countries, low-resourced countries have not been spared the global challenges caused by non-communicable diseases (NCDs), such as cardiovascular diseases, coronary heart disease, hypertension, diabetes mellitus, and obesity [37]. For countries with minimal health budgets, every effort needs to be made to reduce patients' reliance on costly medical solutions to health problems, including NCDs, for which drugs are often the first line of treatment to reduce the associated morbidity and premature mortality [38]. Prevention efforts within the healthcare system need to take a broader public health approach to motivate people to address their unhealthy lifestyles that result in the development of NCDs. To achieve this, however, Ministries of Health in low-resourced countries needs factual, timely, and reliable NCDs data that can build a comprehensive information system to provide evidence-based decisionmaking and to develop appropriate prevention strategies for the specific population affected by NCDs [39].

All healthcare organizations, including governmental and non-governmental institutions, have their strategies to prevent and control diseases and to maintain the health of their community effectively and efficiently. Likewise, healthcare organizations have a strategy for disease prevention and control to maintain the health of the community. Most healthcare systems rely on accurate, timely, correct, and factual data to make political and administrative decisions $[39,40]$. Moreover, healthcare systems should have a balanced approach in preventing both infectious communicable disease and NCDs, which require factual data at the mega, macro, meso, and micro level of healthcare.

\section{Prevalence of obesity increasing in developing countries}

Evidence shows that the prevalence of overweight and obesity is increasing in Bangladesh, especially among women $[41,42,43,44,45]$. The prevalence of overweight and obesity has been raised from 4 to $16 \%$ during the period between 1996 to 2011 [42], according to Bangladesh Demographic and Health Survey (BDHS) [46].

Of the $17 \%$ of overweight or obese adults in Bangladesh, just $4 \%$ were obese, and obesity rates in Bangladesh are increasing at a slower pace. From 1980 to 2013 obesity rates in adults grew from $2 \%$ to 4\%, and rates in children and adolescents remained at about 1.5\%.When looking at the region, South Asia has seen a steady increase in overweight and obesity since 1980, with the rate of overweight or obese adults rising from $16 \%$ to $21 \%$ in 2013 . South Asia also has the lowest child overweight/obesity prevalence among all regions (6\%) and the lowest child obesity prevalence $(3 \%)$.Within the region, Pakistan has the highest obesity rate for adults (14\%), and Bhutan has the highest obesity rate for children (6\%). South Asia also has the smallest gender gap in adult obesity among all regions (4.8\% male, 5.2\% female) [47].

Approximately 39\% of the global adult population were classified as overweight (Body Mass Index (BMI) 25.0-29.9 $\mathrm{kg} / \mathrm{m} 2$ ) or obese (BMI $>29.9 \mathrm{~kg} / \mathrm{m} 2$ ) in 2014; a doubling since 1975[1]. Whereas the prevalence of obesity was $6.4 \%$ among women and $3.2 \%$ among men in 1975 , it had risen to $14.9 \%$ and $10.8 \%$, respectively by 2014 [1]. In developing countries like India, the increasing prevalence of overweight and obesity has coincided with the demographic and epidemiological transitions, in which mortality and fertility have declined, and lifestyle-related diseases have become more common $[48,49,50]$.

The prevalence of overweight and obesity in India is increasing faster than the world average. For instance, the prevalence of overweight increased from $8.4 \%$ to 15.5\% among women between 1998 and 2015, and the prevalence of obesity increased from $2.2 \%$ to $5.1 \%$ over the same period [51]. This fast-paced growth has been accompanied by notable increases in the burden of non-communicable diseases (NCDs).

\section{Conclusion}

Evidence is strongly suggestive that obesity, through pro-inflammatory pathways, predisposes to the development of psoriasis and that obesity aggravates existing psoriasis. While larger, randomized trials are needed to fully elucidate the potential effect of weight reduction on the severity of psoriasis. Unhealthy, processed food has become much more accessible in developing countries food markets. This, combined with rising middle class incomes, is increasing the average caloric intake per individual among middle class and high income households. While obesity is gradually increasing in developing countries, where NCD is yet to implement. If proper NCD program is not implement in right time, severity of psoriasis will increase with increase prevalence of obesity. 


\section{Compliance with ethical standards}

\section{Acknowledgments}

The assistance provided by Mr Reasad Azim was greatly appreciated.

\section{Disclosure of conflict of interest}

No conflict of interest.

\section{References}

[1] Pedersen JK, Svendsen AJ, Hørslev-Petersen K: Incidence of rheumatoid arthritis in the southern part of Denmark from 1995 to 2001. Open Rheumatol J. 2007; 1: 18-23.

[2] Patterson CC, Dahlquist GG, Gyürüs E, Green A, Soltész G: Incidence trends for childhood type 1 diabetes in Europe during 1989-2003 and predicted new cases 2005-20: a multicentre prospective registration study. Lancet. 2009; 373: 2027-2033.

[3] Icen M, Crowson CS, McEvoy MT, Dann FJ, Gabriel SE, Maradit Kremers H: Trends in incidence of adult-onset psoriasis over three decades: a population-based study. J Am Acad Dermatol. 2009; 60: 394-401.

[4] Flegal KM: Prevalence and trends in obesity among US adults, 1999-2008. JAMA. 2010; 303: 235.

[5] World Health Organization: Overweight/obesity: overweight by country. Global Health Observatory Data Repository 2008-2013. Geneva, WHO. 2013.

[6] Samaras K, Kelly PJ, Chiano MN, Spector TD, Campbell LV: Genetic and environmental influences on total-body and central abdominal fat: the effect of physical activity in female twins. Ann Intern Med. 1999; 130: 873-882.

[7] Lindegård B: Diseases associated with psoriasis in a general population of 159,200 middle-aged, urban, native Swedes. Dermatologica. 1986; 172: 298-304.

[8] Eckel RH, York DA, Rössner S, Hubbard V, Caterson I, St. Jeor ST, et al. Prevention Conference VII: Obesity, a Worldwide Epidemic Related to Heart Disease and Stroke: Executive Summary. Circulation. 2004; 110: 29682975.

[9] Popkin BM. The Nutrition Transition and Obesity in the Developing World. J Nutr. 2001; 131: 871S-873S.

[10] WHO. Obesity and overweight. 2016.

[11] Biswas T, Garnett SP, Pervin S, Rawal LB. The prevalence of underweight, overweight and obesity in Bangladeshi adults: Data from a national survey. Nugent RA, editor. PLOS ONE. 2017; 12: e0177395.

[12] Siddiquee T, Bhowmik B, Da Vale Moreira NC, Mujumder A, Mahtab H, Khan AKA, et al. Prevalence of obesity in a rural Asian Indian (Bangladeshi) population and its determinants. BMC Public Health. 2015; 15: 860.

[13] The SuRF Report 2. World Health Organization.

[14] Siddiquee T, Bhowmik B, Karmaker RK, Chowdhury A, Mahtab H, Azad Khan AK, et al. Association of general and central obesity with diabetes and prediabetes in rural Bangladeshi population. Diabetes Metab Syndr Clin Res Rev. 2015; 9: 247-251.

[15] https://worldpopulationreview.com/countries/bangladesh-population

[16] Cao H: Adipocytokines in obesity and metabolic disease. J Endocrinol. 2014; 220: T47-T59.

[17] Gómez R, Conde J, Scotece M, Gómez-Reino JJ, Lago F, Gualillo O: What's new in our understanding of the role of adipokines in rheumatic diseases? Nat Rev Rheumatol. 2011; 7: 528-536.

[18] Miller IM, Ellervik C, Yazdanyar S, Jemec GBE: Meta-analysis of psoriasis, cardiovascular disease.

[19] Versini M, Jeandel P-Y, Rosenthal E, Shoenfeld Y: Obesity in autoimmune diseases: not a passive bystander. Autoimmun Rev. 2014; 13: 981-1000.

[20] Zamboni S, Zanetti G, Grosso G, Ambrosio GB, Gozzetti S, Peserico A: Dietary behaviour in psoriatic patients. Acta Derm Venereol Suppl (Stockh). 1989; 146: 182-183. 
[21] Herron MD, Hinckley M, Hoffman MS, Papenfuss J, Hansen CB, Callis KP, et al: Impact of obesity and smoking on psoriasis presentation and management. Arch Dermatol. 2005; 141: 1727-1534.

[22] Versini M, Jeandel P-Y, Rosenthal E, Shoenfeld Y: Obesity in autoimmune diseases: not a passive bystander. Autoimmun Rev. 2014; 13: 981-1000.

[23] Michalak-Stoma A, Pietrzak A, Szepietowski JC, Zalewska-Janowska A, Paszkowski T, Chodorowska G: Cytokine network in psoriasis revisited. Eur Cytokine Netw. 2011; 22: 160-168.

[24] Zhu K-J, Zhang C, Li M, Zhu C-Y, Shi G, Fan Y-M: Leptin levels in patients with psoriasis: a meta-analysis. Clin Exp Dermatol. 2013; 38: 478-483.

[25] Bulló M, García-Lorda P, Megias I, Salas-Salvadó J: 25. Systemic inflammation, adipose tissue tumor necrosis factor, and leptin expression. Obes Res. 2003; 11: 525-531.

[26] Johnston A, Arnadottir S, Gudjonsson JE, Aphale A, Sigmarsdottir AA, Gunnarsson SI, et al: Obesity in psoriasis: leptin and resistin as mediators of cutaneous inflammation. Br J Dermatol. 2008; 159: 342-350.

[27] Coimbra S, Oliveira H, Reis F, Belo L, Rocha S, Quintanilha A, et al: Circulating adipokine levels in Portuguese patients with psoriasis vulgaris according to body mass index, severity and therapy. J Eur Acad Dermatol Venereol. 2010; 24: 1386-1394.

[28] Takahashi H, Tsuji H, Honma M, Ishida-Yamamoto A, Iizuka H: Increased plasma resistin and decreased omentin levels in Japanese patients with psoriasis. Arch Dermatol Res. 2013; 305: 113-116.

[29] Kawashima K, Torii K, Furuhashi T, Saito C, Nishio E, Nishida E, et al: Phototherapy reduces serum resistin levels in psoriasis patients. Photodermatol Photoimmunol Photomed. 2011; 27: 152-155.

[30] Çerman AA, Bozkurt S, Sav A, Tulunay A, Elbaşı MO, Ergun T: Serum leptin levels, skin leptin and leptin receptor expression in psoriasis. Br J Dermatol. 2008; 159: 820-826.

[31] Jin Y, Zhang F, Yang S, Kong Y, Xiao F, Hou Y, et al: Combined effects of HLA-Cw6, body mass index and waist-hip ratio on psoriasis vulgaris in Chinese Han population. J Dermatol Sci. 2008; 52: 123-129.

[32] Armstrong AW, Harskamp CT, Armstrong EJ. The association between psoriasis and obesity: a systematic review and meta-analysis of observational studies. Nutr Diabetes. 3 Dec 2012; 2: e54.

[33] Kanemaru K, Matsuyuki A, Nakamura Y, Fukami K. Obesity exacerbates imiquimod-induced psoriasis-like epidermal hyperplasia and interleukin-17 and interleukin-22 production in mice. Exp Dermatol. 2015; 24: 43642.

[34] Dalamaga M. Adipocytokines and psoriasis: insights into mechanisms linking obesity and inflammation to psoriasis. World J Dermatol. 2013; 2: 27.

[35] Upala S, Sanguankeo A. Effect of lifestyle weight loss intervention on disease severity in patients with psoriasis: a systematic review and meta-analysis. Int J Obes. 2015; 39: 1197-202.

[36] Kyriaki Paroutoglou, Evangelia Papadavid, Gerasimos Socrates Christodoulatos \& Maria Dalamaga; Deciphering the Association Between Psoriasis and Obesity: Current Evidence and Treatment Considerations; Current Obesity Reports. 2020; 9: 165-178.

[37] Kassa MD, Grace J. Healthcare professionals' perceptions of non-communicable diseases risk factors and its regional distribution in Ethiopia. Global Journal of Health Science. 2018; 10(1): 88-97.

[38] Justine F, Davies I, Wagner RG. Weighing up the costs of treating 'lifestyle' diseases in South Africa. University of the Witwatersrand. 2019; 24(3): 1-13.

[39] Melkamu Dugassa Kassa JMG. A mixed-method study of quality, availability and timeliness of non- communicable disease (NCD) related data and its link to NCD prevention: Perceptions of health care workers in Ethiopia. Health Information Management Journal. 2018; 47(4): 21-34.

[40] Anwar MS, Id AGJ, Id NLM. Heart failure and healthcare informatics. PLoS Medicine. 2019; 16(5): 4-7.

[41] Sarma H, Saquib N, Hasan MM, Saquib J, Rahman AS, Khan JR, Uddin MJ, Cullen MR, Ahmed T. Determinants of overweight or obesity among ever-married adult women in Bangladesh. BMC Obesity. 2016; 3(1): 13. [PMC free article] [PubMed] [Google Scholar] 
[42] Hoque ME, Long KZ, Niessen LW, Mamun AA. Rapid shift toward overweight from double burden of underweight and overweight among Bangladeshi women: a systematic review and pooled analysis. Nutr Rev. 2015; 73(7): 438-447. [PubMed] [Google Scholar]

[43] Kamal SM, Hassan CH, Alam GM. Dual burden of underweight and overweight among women in Bangladesh: patterns, prevalence, and sociodemographic correlates. J Health Popul Nutr. 2015; 33(1): 92. [PMC free article] [PubMed] [Google Scholar]

[44] Khan MM, Krämer A. Factors associated with being underweight, overweight and obese among ever-married non-pregnant urban women in Bangladesh. Singap Med J. 2009; 50(8): 804. [PubMed] [Google Scholar]

[45] Shafique S, Akhter N, Stallkamp G, de Pee S, Panagides D, Bloem MW. Trends of under-and overweight among rural and urban poor women indicate the double burden of malnutrition in Bangladesh. Int J Epidemiol. 2007; 36(2): 449-457.

[46] The DHS Program. Demographic and Health Surveys.

[47] Stewart R, Persaud M; Adult rates of overweight and obesity rise of Bangladesh; Institute for health metrics and evaluation: University of Washington; Newsletter.

[48] Tandon N. et al. The increasing burden of diabetes and variations among the states of India: the Global Burden of Disease Study 1990-2016. Lancet Glob. Heal. 2018; 6: e1352-62.

[49] Dandona L. et al. Nations within a nation: variations in epidemiological transition across the states of India, 19902016 in the Global Burden of Disease Study. Lancet. 2017; 390: 2437-60.

[50] Prabhakaran D. et al. The changing patterns of cardiovascular diseases and their risk factors in the states of India: the Global Burden of Disease Study 1990-2016. Lancet Glob. Heal. 2018; 6: e1339-51.

[51] International Institute for Population Sciences. National Family Health Survey (NFHS-4) 2015-16 India. International Institute for Population Sciences (IIPS) and ICF. 2017. 